Scholz, Joachim

\title{
"In zweifelhaften Fällen mag der Geist der Milde den Ausschlag geben". Korrektur und Benotung des deutschen Abituraufsatzes in historischen Debatten und Praktiken
}

Reh, Sabine [Hrsg.]; Bühler, Patrick [Hrsg.]; Hofmann, Michèle [Hrsg.]; Moser, Vera [Hrsg.]: Schülerauslese, schulische Beurteilung und Schülertests 1880-1980. Bad Heilbrunn : Verlag Julius Klinkhardt 2021, S. 153-170. - (Bildungsgeschichte. Forschung - Akzente - Perspektiven)

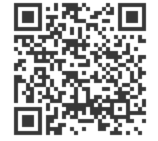

\section{Quellenangabe/ Reference:}

Scholz, Joachim: "In zweifelhaften Fällen mag der Geist der Milde den Ausschlag geben". Korrektur und Benotung des deutschen Abituraufsatzes in historischen Debatten und Praktiken - In: Reh, Sabine [Hrsg.]; Bühler, Patrick [Hrsg.]; Hofmann, Michèle [Hrsg.]; Moser, Vera [Hrsg.]: Schülerauslese, schulische Beurteilung und Schülertests 1880-1980. Bad Heilbrunn : Verlag Julius Klinkhardt 2021, S. 153-170 - URN: urn:nbn:de:0111-pedocs-222757 - DOI: 10.25656/01:22275

https://nbn-resolving.org/urn:nbn:de:0111-pedocs-222757

https://doi.org/10.25656/01:22275

in Kooperation mit / in cooperation with:

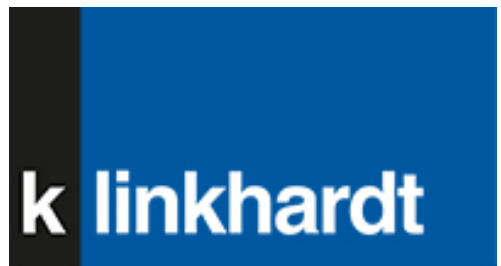

http://www.klinkhardt.de

\section{Nutzungsbedingungen}

Dieses Dokument steht unter folgender Creative Commons-Lizenz: http://creativecommons.org/licenses/by-nc-sa/4.0/deed.de - Sie dürfen das Werk bzw. den Inhalt unter folgenden Bedingungen vervielfältigen, verbreiten und öffentlich zugänglich machen sowie Abwandlungen und Bearbeitungen des Werkes bzw. Inhaltes anfertigen: Sie müssen den Namen des Autors/Rechteinhabers in der von ihm festgelegten Weise nennen. Dieses Werk bzw. der Inhalt darf nicht für kommerzielle Žwecke verwendet werden. Die neu entstandenen Werke bzw. Inhalte dürfen nur unter Verwendung von Lizenzbedingungen weitergegeben werden, die mit denen dieses Lizenzvertrages identisch oder vergleichbar sind.

Mit der Verwendung dieses Dokuments erkennen Sie die Nutzungsbedingungen an.

\section{Terms of use}

This document is published under following Creative Commons-License: http://creativecommons.org/licenses/by-nc-sa/4.0/deed.en - You may copy, distribute and transmit, adapt or exhibit the work in the public and alter, transform or change this work as long as you attribute the work in the manner specified by the author or licensor. You are not allowed to make commercial use of the work. If you alter, transform, or change this work in any way, you may distribute the resulting work only under this or a comparable license.

By using this particular document, you accept the above-stated conditions of use.

\section{Kontakt / Contact:}

peDOcs

DIPF | Leibniz-Institut für Bildungsforschung und Bildungsinformation

Informationszentrum (IZ) Bildung

E-Mail: pedocs@dipf.de

Internet: www.pedocs.de

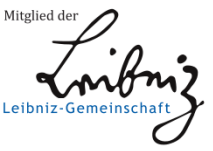


Bildungsgeschichte.

Forschung - Akzente - Perspektiven

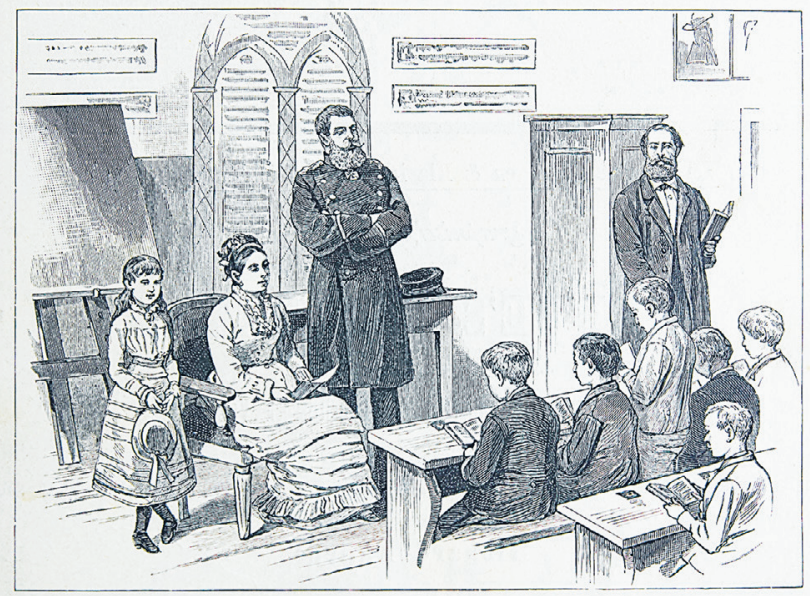

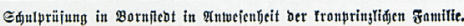

Sabine Reh / Patrick Bühler Michèle Hofmann/Vera Moser (Hrsg.)

\section{Schülerauslese,} schulische Beurteilung und Schülertests 1880-1980 


\section{Sabine Reh \\ Patrick Bühler \\ Michèle Hofmann \\ Vera Moser \\ (Hrsg.)}

\section{Schülerauslese, schulische Beurteilung und Schülertests 1880-1980}


Dieser Titel wurde in das Programm des Verlages mittels eines Peer-Review-Verfahrens aufgenommen. Für weitere Informationen siehe www.klinkhardt.de.

Bibliografische Information der Deutschen Nationalbibliothek Die Deutsche Nationalbibliothek verzeichnet diese Publikation in der Deutschen Nationalbibliografie; detaillierte bibliografische Daten sind im Internet abrufbar über http://dnb.d-nb.de.

2021.1. (C) by Julius Klinkhardt.

Abbildung Umschlag: Schulprüfung in Bornstedt, Quellenangabe: Daheim. Ein deutsches Familienblatt mit Illustrationen 19 (1883) 3, Beil. 2.

Druck und Bindung: AZ Druck und Datentechnik, Kempten.

Printed in Germany 2021.

Gedruckt auf chlorfrei gebleichtem alterungsbeständigem Papier.

Die Publikation (mit Ausnahme aller Fotos, Grafiken und Abbildungen) ist veröffentlicht unter der Creative Commons-Lizenz: CC BY-NC-SA 4.0 International https://creativecommons.org/licenses/by-nc-sa/4.0/ 


\section{Inhaltsverzeichnis}

Sabine Reh, Patrick Bühler, Michèle Hofmann und Vera Moser

Einleitung

Prüfen, Testen, Auslesen und Zuweisen. Zum Inklusions-Paradox

des Schulsystems

Jona Garz, Vera Moser und Stefan Wünsch

Die „Kielhorn-Rede“: Ursprungsmythos der deutschen Hilfsschule

Jona Garz

„Schriftproben von schwachsinnigen resp. idiotischen Kindern“.

Testwissen zwischen Psychiatrie und Pädagogik um 1900

\section{Michèle Hofmann}

Grenzziehungen - Praktiken der Kategorisierung geistig

„anormaler“ Kinder um 1900 in der Schweiz

\section{Patrick Bühler}

„Komplett pessimistisch eingestellt“. Hilfe und Heilung in der

Schweizer Sonderpädagogik zu Beginn des 20. Jahrhunderts

Cristina Alarcón López

Genealogie des Grundschulgutachtens im Zeichen des Dispositivs der

„Schülerauslese“

\section{Rebecca Heinemann}

Im „Mittelpunkt sowohl der theoretisch-psychologischen wie der angewandt psychologischen Arbeit“. Das personalistische Begabungskonzept William Sterns

Susanne Schregel

„Übernormalen-Pädagogik“ und „Begabtenschulen“ zwischen

Kaiserreich und Weimarer Republik

\section{Joachim Scholz}

„In zweifelhaften Fällen mag der Geist der Milde den Ausschlag geben“-

Korrektur und Benotung des deutschen Abituraufsatzes in historischen

Debatten und Praktiken 
Kerrin v. Engelhardt

„Der papierene Drache“ - Der Reifeprüfungsaufsatz zwischen

1890 und 1930

Thomas Hoffmann

Übungsschulen für „Gehirnkrüppel“: Diagnostik, Therapie und heilpädagogische Behandlung hirnverletzter Soldaten 1914-1918

\section{Johanna Lerch}

„Das Kind vor verfehlter Wahl geschützt“. Die Einführung eines „berufspsychologischen Schülerbeobachtungsbogens" in Berliner Schulen, 1917-1923

\section{Fanny Isensee}

„Intelligence tests were given in order to obtain a basis for classifying the pupils" - Die Reclassification Projects in New York City in den 1920er Jahren

Nadja Wenger

„Ihr gebt mich fort, weil ihr mich nicht gern habt." Gutachten der St. Galler Fürsorgestelle für Anormale in den 1940er-Jahren

Michaela Vogt

Das Hilfsschulaufnahmeverfahren als „Grenzzone“ der

Schülerauslese in BRD und DDR

Autor*innenangaben 


\section{„In zweifelhaften Fällen mag der Geist der Milde den Ausschlag geben" - Korrektur und Benotung des deutschen Abituraufsatzes in historischen Debatten und Praktiken}

Bilanziert man die großen Linien bildungs- und schulgeschichtlicher Forschung der letzten vier Jahrzehnte, war darin die Auseinandersetzung mit Systemfragen sowohl der Organisation als auch des im Schulwesen beschäftigten Personals bestimmend. Das zeigen zuallererst die publizistischen Großprojekte wie das Handbuch oder die Datenhandbücher zur Deutschen Bildungsgeschichte, aber auch zahlreiche Einzelstudien aus der sozialhistorischen Forschung. Dabei konnten, wie Kluchert in einem Beitrag über die Geschichte der Gymnasiallehrer seit der Kaiserzeit herausgearbeitet hat, vielfältige Erkenntnisse über die soziale und ökonomische Lage sowie die Professionalisierung des Berufsstandes, oder aber über seinen Standpunkt in den wechselnden politischen Systemen der letzten 150 Jahre gesammelt werden (Kluchert 2014). Die konkrete Arbeit von Lehrerinnen und Lehrern sowie ihr Selbstverständnis jenseits kollektivbiografischer Lagen sind dagegen Bereiche, in denen im Ergebnis der Zusammenschau von wenigen vorliegenden Publikationen noch immer größere Forschungsdesiderate bestehen. ${ }^{1}$ Gerade im Hinblick auf das alltägliche berufliche Tun fällt auf, dass bis in neueste Zeit historisch-empirische Studien selten sind, selbst wenn es sich um Kernbereiche des professionellen Handelns - um das Prüfen etwa, das Selektieren oder das Disziplinieren - handelt. Ricken und Reh vermuten, dass diese Aspekte von der bildungshistorischen Forschung nicht zufällig außer Acht gelassen werden, vertragen sie sich doch weniger mit hergebrachten Narrativen guter Pädagogik (Ricken \& Reh 2017, 247). Dabei dürfte es kaum einen geeigneteren Gegenstand als die Geschichte der Prüfung geben, um Einblicke in die Praxisformen des Schulischen zu gewinnen, die im Modernisierungsprozess des 19. und 20. Jahrhunderts Struktur annahmen und schulische Kulturen nachhaltig gestalteten. Von dieser Annahme ausgehend, wurden im Forschungsprojekt „Abiturprüfungspraxis und Abituraufsatz 1882 bis 1972" die wichtigste schulische Prüfung in Deutschland und ihr für lange Zeit jedenfalls zentraler Bestandteil, der deutsche Auf-

1 Die Aufzählung relevanter Forschungsarbeiten ebd., S. 36. Eine kurze treffende Bilanz über die Historische Bildungsforschung und ihre Forschungsdefizite zieht auch Jacobi 2007. 
satz, in den Blick genommen. ${ }^{2}$ Im Zentrum des Interesses standen die Rolle und Funktion einer Prüfungsform, die im meritokratisch legitimierten Schulsystem ein spezifisches Anforderungsprofil aufweist, das einerseits pädagogische Erwartungen beflügelte wie es anderseits früh im Verdacht stand, der Privilegierung herrschender Schichten Vorschub zu leisten (Kämper-van den Boogaart 2012). Im folgenden Beitrag werden nach dem kurzen Umriss der Entwicklungslinien im Prüfungswesen und der Rolle des deutschen Abituraufsatzes fallweise Diskursund Praxisformen betrachtet, die die Besonderheiten und die perrenierende Problematik der Prüfungsform des deutschen Aufsatzes zum einen in der Reflexion preußischer Lehrkräfte um 1900 und zum anderen im alltäglichen Prozess ihrer Prüfungspraxis zu zwei unterschiedlichen Zeitpunkten zeigen.

\section{Prüfungen und deutscher Aufsatz in der Schulgeschichte Deutschlands}

Die Entwicklung eines elaborierten Prüfungswesens war sehr grundlegend eingebunden in den gesamtgesellschaftlichen Prozess der Durchsetzung meritokratischer Standards, dem die Schule nicht nur unterworfen war, sondern den sie auch selbst antrieb und mitgestaltete (Berdelmann u.a. 2018). Allgemeine Züge der Herausbildung moderner schulischer Prüfungsformen waren die Zurückdrängung diffuser Aspekte und partikularer Zwecksetzungen. So verschwand die im 18. Jahrhundert übliche Form der Prüfung, die als öffentliche Präsentation anlässlich von Schulfeiern sowohl den Schülern als auch nicht unmaßgeblich ihren Lehrern galt. Auch die Beurteilung von Fleiß und Tugend oder die Dokumentation bloßer Leistungsfortschritte schwanden mehr und mehr aus dem Zielfokus der schulischen Prüfung (Reh u.a. 2017).

Unter den einschränkenden Bedingungen des noch bis ins 20. Jahrhundert hinein bestehenden „sozialen Klassenschulwesens“ (Müller 1977) und der funktionalen Differenz zwischen dem höheren und dem niederen Schulwesen kam es zuerst an den Gymnasien zu einer Meritokratisierung der schulischen Prüfung. Mehr und mehr ging es nun um eine als objektiv geltende Feststellung von Lernleistungen. Dementsprechend erfolgte der Entwurf von Bildungsangeboten in unterschiedlichen Klassen, Schulen und Schulformen, auch unter Berücksichtigung individueller Begabungsunterschiede. Teilweise erfolgte auch in der Volksschule eine rangmäßige Platzierung der Schüler*innen in der Sitzordnung der Klasse und ein Ausweis ihres Platzes auf Jahresendzeugnissen. Doch die biografisch folgenreiche

2 Das historisch-praxeologische Forschungsprojekt unter der Leitung von Sabine Reh, Michael Kämper-van den Boogaart und Joachim Scholz ist 2016 aus dem Leibniz-Wettbewerb hervorgegangen. Die reguläre dreijährige Laufzeit endete 2019. 
Kanalisierung der Schüler*innen in den zu unterschiedlichen Berechtigungen führenden Zweigen des Schulwesens entfaltete ihre Dynamik zuerst an den höheren Schulen. Besondere Relevanz erlangte dabei die gymnasiale Abschlussprüfung, das Abitur bzw. die Matura (Bölling 2010; Jeismann 1996).

Als zentrale Scharnierstelle des Berechtigungswesens regelte diese Prüfung in zunehmend verbindlicher Weise den Übergang vom Gymnasium zur Universität. Ein erstes Abitureglement war 1788 in Preußen erlassen worden. Die vom Rektor des Friedrichswerderschen Gymnasiums in Berlin, Friedrich Gedike (17541803), ausgearbeitete Verordnung hat vor allem aufgrund ihrer formalen Anregerfunktion für die künftige Rolle des Abiturs Gewicht. Die Festlegungen waren hier noch sehr unverbindlich; inhaltlich blieb das Abiturreglement gänzlich unspezifisch. Die Abiturprüfungserlasse von 1788 und 1812 regelten vor allem Anforderungen der Universitätsseite und die Vergabe von Stipendien. Erst 1834 wurde das Abitur in Preußen und den meisten Staaten des Deutschen Bundes verpflichtende Voraussetzung für die Immatrikulation. Zwischenstaatlich bestanden allerdings sehr unterschiedliche Regelungen. Wurden etwa in Preußen die Inhalte der Abiturprüfungen einzelschulisch ausgemacht, so war in Bayern bereits seit der Mitte des 19. Jahrhunderts das Prüfungsverfahren zentral geregelt. Daraus resultierten Differenzen, die die gesamte Verwaltung des Verfahrens, von der Aufgabenstellung bis zur Normierung der Korrekturvorgaben, betrafen. Unterschiedliche Prüfungskulturen ergaben sich freilich auch aus der fachkulturellen Logik einzelner Unterrichtsgebiete sowie aus den Konjunkturen einzelner Prüfungsverfahren.

Der in den preußischen Prüfungsordnungen seit 1812 besonders ausgewiesene deutsche Aufsatz stand durchaus für eine eigene Richtung. Für sehr lange Zeit hatte er im Kanon der abiturrelevanten Prüfungsgegenstände als Teil der schriftlichen Prüfung eine herausgehobene Position inne, die er erst mit den Strukturreformen im Bildungswesen der Bundesrepublik in den 1970er-Jahren einbüßte. Seine besondere Stellung resultierte aus der unter Schulmännern verbreiteten Überzeugung, dass sich an keinem anderen Gegenstand der Grad der „Bildung“ eines Schülers besser ermitteln ließe als an ihm. In den preußischen Verordnungen findet die Idee, aus dem deutschen Aufsatz könne man mehr als nur „Kenntniß der deutschen Sprache und die Gewandtheit in deren Gebrauch" (Schultze 1831, 14) ablesen, schon 1812 Niederschlag. Der Prüfling solle im deutschen Aufsatz „vorzüglich die Bildung des Verstandes und der Phantasie beurkunden“ (ebd.). In der noch stärker auf den Allgemeinbildungsgedanken akzentuierenden Prüfungsordnung von 1834 wird diese Formulierung übernommen und die Rolle des deutschen Aufsatzes dann dadurch näher bestimmt, dass an ihm die "Gesammtbildung des Examinanden“" ablesbar sein sollte (Neigebaur 1835, 214).

Von der Preußischen Schulverwaltung wurde der deutsche Aufsatz damals mit dem Gedanken einer über Fachgrenzen hinweggehenden Beachtung der eigenen „Tüchtigkeit“ verknüpft, die zur selben Zeit auch die Ausprägung anderer 
Arrangements, wie z.B. die Organisation von Schulklassen als Jahrgangsklassen, beeinflusste (Scholz \& Reh 2016). So hatte das Königliche Schulkollegium der Provinz Brandenburg 1829 verfügt, dass bei der abschließenden Einschätzung der Befähigungen eines Schülers immer auch in Erwägung zu ziehen sei, „ob er in dem ihm aufgegebenen deutschen Aufsatz dargethan habe, daß er einen seinem Fassungsvermögen angemessenen Gegenstand klar und wohlgeordnet [...] vorzutragen wisse [...] und durch die ermittelte Gesammtbildung einen verhältnismäßigen Grad von Mangel an speciellen Kenntnissen zu compensiren“ in der Lage sei (Schultze 1831, 106).

Künftige prüfungspraktische Regelungen folgten der starken Gewichtung des deutschen Aufsatzes durch Ausdehnung der ihm eingeräumten Prüfungszeit sowie auch der Relevanz der Prüfungsresultate. Nur im Deutschen Aufsatz wurde Schülern eine Bearbeitungszeit von fünf Stunden eingeräumt, für alle anderen schriftlichen Prüfungen, außer Latein, standen zwei bis vier Stunden zur Verfügung. Im Laufe der Zeit erhielt der deutsche Aufsatz dann einen noch exponierteren Stellenwert. Seit 1882 konnte eine nicht bestandene Prüfung im Abitur „durch mindestens gute Leistungen in einem anderen obligathorischen wissenschaftlichen Gegenstande als ergänzt [d.h. ausgeglichen, JS] erachtet werden " (Cirkularerlass 1882, 376). Zehn Jahre später erhielt diese Regelung eine markante Einschränkung. Nun heißt es in der preußischen Reifeprüfungsordnung: „Bei nicht genügenden Gesammtleistungen im Deutschen oder in den beiden alten Sprachen darf das Reifezeugnis überhaupt nicht ertheilt werden " (Ordnung der Reife- und Abschlußprüfungen 1892, 291. Hervorhebung im Original). Formal gesehen erhielt dadurch der Deutschaufsatz eine Vorrangstellung unter den Abiturprüfungen: Wer ihn nicht bestand, konnte das Abitur insgesamt nicht erringen. Dieser dann nur wenige Jahre geltenden Regelung ${ }^{3}$ unmittelbar vorangegangen war die Schulkonferenz von 1890, auf der Kaiser Wilhelm II. in seiner Eröffnungsansprache gegen die Alten Sprachen das Wort erhoben und den Bildungswert des deutschen Unterrichts und des Aufsatzes besonders betont hatte:

Wir müssen als Grundlage für das Gymnasium das Deutsche nehmen; wir sollen nationale junge Deutsche erziehen und nicht junge Griechen und Römer. Wir müssen von der Basis abgehen, die jahrhundertelang bestanden hat, von der alten klösterlichen Erziehung des Mittelalters, wo das Lateinische maßgebend war und ein bißchen Griechisch dazu. Das ist nicht mehr maßgebend, wir müssen das Deutsche zur Basis machen. Der Deutsche Aufsatz muß der Mittelpunkt sein, um den sich alles dreht. Wenn einer im Abiturientenexamen einen tadellosen deutschen Aufsatz liefert, so kann man daraus das Maß der Geistesbildung des jungen Mannes erkennen und beurteilen, ob er etwas taugt oder nicht (Michael \& Schepp 1973, 416).

3 In der Ordnung der Reifeprüfung von 1901 wird dieses Alleinstellungsmerkmal nach Kritik wieder aufgehoben. 
Nicht zuletzt diese von höchster Stelle intendierte Aufwertung des nationalen Elementes war es, durch die der deutsche Aufsatz in der Forschung immer wieder charakterisiert wurde. Wie im Folgenden gezeigt werden soll, befassten sich Protagonisten der Schulwelt in regen Diskussionen um den deutschen Aufsatz vorrangig aber mit anderen Problemen. Schulpraktiker selbst beklagten einerseits im besagten Zeitraum eher das hohe Gewicht des Aufsatzes als Prüfungsmittel, denn wer von ihnen wollte die Verantwortung für das Scheitern eines Schülers im Abitur tragen, weil dieser den deutschen Aufsatz nicht bestanden hatte. Die Prüfungspraxis warf darüber hinaus Fragen der Korrektur und Benotung auf, die ein Licht auf die Probleme der Prüfungsform deutscher Aufsatz warfen, aber auch das Problembewusstsein der Professionellen und deren Lösungsvorstellungen kenntlich machen.

\section{Die Debatte um Korrektur und Benotung des deutschen Aufsatzes}

Auf eine erstaunliche Breite überlieferter Publizistik zu den Fragen um die Gestaltung der Prüfungsform im deutschen Aufsatz konnten schulische Akteure bereits an der Schwelle zum 20. Jahrhundert zugreifen. Debatten über den deutschen Aufsatz wurden auf Direktorenkonferenzen, in Schulprogrammen, in Zeitschriften der Schulmänner und in einer großen Zahl besonderer Schriften geführt. ${ }^{4}$ Ein Bewusstsein dafür, dass der deutsche Aufsatz trotz oder gerade auch wegen der hohen Ansprüche, die sich mit ihm verbanden, eigene Problemlagen erzeugte, war bereits im 19. Jahrhundert entstanden. Seit den 1830er-Jahren spiegeln preußische Ministerialreskripte und Verfügungen, so rekonstruierte bereits Apelt (1907), die Auffassung, dass Themen, „bei welchen der Schüler über ganz abstrakte oder ihm unbekannte Gegenstände sogenannte eigene Gedanken produzieren soll, ... die Grenzen des Gymnasialunterrichts" überschreiten würden. Sie seien „unzweckmäßig und gereichen dem Lehrer, der sie stellt, mit Recht zum Vorwurf, und dem Schüler, der sie bearbeiten soll, zur Qual“ (ebd., 27). Als die beste Möglichkeit, solchen Auswüchsen zu wehren, wurde vielfach empfohlen, den Aufsatz in engere Verbindung mit dem Unterricht zu bringen. Die dabei in Vorschlag stehenden Erwägungen liefen darauf hinaus, entweder andere Unterrichtsfächer verstärkt in den Dienst des deutschen Aufsatzes zu stellen, oder den Deutschun-

4 Ein wichtiger Publikationsort von Stellungnahmen zum deutschen Aufsatz war die seit 1883 erscheinende Zeitschrift „Gymnasium“, in der Schulpraktiker sich der Problematik des Deutschen Aufsatzes in großer Regelmäßigkeit annahmen, entweder in dezidierten Beiträgen, in Rezensionen oder auch in der Rubrik „Programmschau“, für die dann selbst Veröffentlichungen von Lehrern in den jährlichen Schulprogrammen der höheren Lehranstalten aufgestellt und inhaltlich ausgewertet wurden. 
terricht selbst inhaltlich so auszugestalten, dass aus ihm anregungsreiche Aufsatzstoffe gewonnen werden könnten. Galt es einerseits, den Deutschlehrer in größere Nähe zu anderen Unterrichtsgegenständen zu bringen und dabei auf „Einheit der Behandlung" (ebd., 29) zu achten, so ging es andererseits darum, z.B. durch die Verwendung von Klassikerschriften einen stofflichen Kanon zu generieren, der die stärkere Gründung der Aufsatzinhalte im Unterricht zuließ.

Eine besonders bemerkenswerte Zusammenstellung der Abhandlungen einer preußischen Direktorenkonferenz in der Provinz Sachsen legte 1899 Gustav Legerlotz (1832-1904), Rektor eines preußischen Gymnasiums, zur Frage „Nach welchen Gesichtspunkten ist der deutsche Aufsatz in den oberen Klassen zu wählen, vorzubereiten und zu korrigieren" vor (Legerlotz 1899). Mit der nachträglichen Veröffentlichung dieses über 100 Seiten starken Konvoluts (Legerlotz 1900) ging Legerlotz weit über Vorläufer hinaus, wie sie auch aus anderen Direktorenversammlungen vorlagen (vgl. etwa Hense \& Meyer 1882 für Westfalen oder für Posen Schröer \& Dolega 1891). Solche Konferenzprotokolle zeigen souveräne Akteure im professionellen Diskurs auf der Grundlage der aktuellen Verordnungslage sowie im Bewusstsein darüber, dass ihre Diskussionen Einfluss auf die Gestaltung und Verbesserung der behördlichen Verfügungen haben werden. ${ }^{5}$ Ihr besonderer Quellenwert resultiert zweifellos aus der Praxisnähe der wiedergegebenen Stellungnahmen - sie waren Instrumente der Reflexion über und zur Gestaltung der Praxis - wobei die Quelle von 1899 noch dadurch hervorsticht, dass sie in die Zeit der gesteigerten Bedeutung des Aufsatzes als Prüfungsgegenstand fällt und die Frage schulischer Selektivität, die im höheren Schulwesen sonst nachrangig kursierte, vielen Referenten wenigstens theoretisch greifbar vor Augen stand. Ein wiederkehrender Grundgedanke in den Aussagen der Philologen, die allesamt aus eigenen Prüfungserfahrungen referieren, ${ }^{6}$ besteht darin, die zahlreichen diffusen Anteile der Aufsatzleistung in den Griff zu bekommen und Klarheit über die Anforderungen zu schaffen, denen die Schüler im deutschen Aufsatz genügen sollten. Das war auch nötig, denn der deutsche Aufsatz im preußischen Gymnasium hatte durch seinen Nimbus als Gradmesser der allgemeinen Bildung einen sehr eigenen Charakter. Unter den Rektoren herrschte weitgehend Konsens darüber, dass in ihm stoffliche Inhalte sehr unterschiedlicher Fächer zum Thema werden konnten und sollten. Auch stand für sie außer Frage, dass im Aufsatz die Grenzen zum außerunterrichtlichen Bereich überschritten wurden; die Themen sollten

5 Direktorenversammlungen fanden jährlich statt. Es standen Thesen auf der Tagesordnung, die verhandelt werden sollten, begleitet wurde dies durch jeweils ein Hauptreferat und ein Gegenreferat. Nach den Referaten folgte die Diskussion, die Verhandlung über die Thesen, die in eine Abstimmung mündete. Diskussionen wurden ausführlich protokolliert. - Zur faktischen Souveränität der Gymnasiallehrer gegenüber und in der Kultusadministration vgl. Jeismann 1999.

6 Zur Frage der damals noch wenig ausdifferenzierten fachlichen Zuordnung und Lehrbefähigung der Philologen vgl. Schubring 1991 und Jeismann 1996, 297-300. 
einen Bezug zum Erfahrungskreis der Schüler haben. Allein schon für die Aufgabenstellung, geschweige denn später für die Beurteilung der Aufsätze als Prüfungsleistungen, resultierte daraus die Schwierigkeit sicherzustellen, dass die Anforderungen in dieser Prüfungsform insgesamt nicht zu hoch oder zu niedrig angesetzt werden. Eine Aufgabenstellung im deutschen Aufsatz eröffnete prinzipiell einen unübersichtlichen Raum von Bearbeitungsmöglichkeiten und barg gerade für pädagogisch wohlmeinende Lehrer Risiken. Legerlotz, als langjährigem Kenner der Praxis, fiel es nicht schwer, Beispiele für Heuchelei und Unwahrhaftigkeit provozierende („Die edelsten Freuden des Jünglings“), für „erkünstelte Gefühl““ einfordernde („Zuruf an die im Herbste vorüberziehenden Störche“), platte („Vergleichung der Lüge mit einem Schneeball") oder solche unangemessene Aufgaben zu finden, die „zu altkluger oder gar greisenhafter Rederei verlocken“ („In den Ozean schifft mit tausend Masten der Jüngling; still auf gerettetem Boot treibt in den Hafen der Greis“) (Legerlotz 1899, 44f.).

Die Überzeugung, dass der deutsche Aufsatz auch die Phantasie der Schüler zum Vorschein bringen sollte, war auf der anderen Seite erwünscht und schon lange ein pädagogisches Postulat, über das leicht Konsens herzustellen war. Die Direktorenversammlung stimmte durchaus darin überein, dass das Abfassen eines Aufsatzes mehr als nur eine reproduktive Tätigkeit sein sollte und über das Buchwissen hinaus persönliche Erfahrungen in den Aufsatz einfließen durften und sollten (Legerlotz 1899, 18). Beflügelt vom Eindringen reformpädagogischer Rhetorik hatten gegen Ende des 19. Jahrhunderts diese schon länger bestehenden Auffassungen noch an Gewicht gewonnen. Angesichts des zeitgleich steigenden Bewusstseins für die Notwendigkeit einer objektiven Beurteilung von Schülerleistungen bildeten diese Postulate jedoch auch ein zusätzliches Einfallstor für diffuse Ansprüche und neue Probleme, die es zu bearbeiten galt.

Vor dem Hintergrund dieser Lage werden vielleicht die Reflexionstiefe und das Fingerspitzengefühl der Schulmänner verständlich, die in ihren Berichten aus der Praxis auch die Folgen und Erschwernisse in der gerechten Beurteilung bedachten, die der deutsche Aufsatz regelmäßig und vor allem dann mitproduzierte, wenn die Themenvorgabe die Grenzen der Unterrichtsinhalte überschritt. Der Referent zitierte zwar selektiv, aber doch so aus den einzelnen Abhandlungen, dass kontroverse Positionen und somit auch der Meinungsbildungsprozess als solcher sichtbar blieben. Deutlich wird zum Beispiel, wie die bevorzugte Stellung des Aufsatzes einerseits das Selbstbewusstsein der mit dem deutschen Aufsatz und seiner Korrektur befassten Lehrer beflügelte, viele von ihnen aber das Postulat, dieser Aufsatz müsse im Mittelpunkt allen Unterrichts in der Schule stehen, als bedrückend empfanden. Die Art der Dokumentation war geeignet, Entlastung darin zu schaffen, wie die Entscheidungsfindung in tatsächlich relevanten Zweifelsfällen im Sinne der Herstellung einer besseren Praxis sich vollziehen kann. Dabei ging es insbesondere darum, den Grad der Unbestimmbarkeit der Schülerleistungen, 
den viele Korrektoren monierten, einzugrenzen. Bewährt hatte sich demnach, die Aufsatzproduktion so weit wie möglich an unterrichtliche Vorbereitungen zu knüpfen. Empfohlen wurde ferner, die einzelnen Bestandteile eines Aufsatzes und die Abfolge der zu seiner Anfertigung nötigen Arbeitsschritte genau zu bestimmen und die jeweiligen Anforderungen zu spezifizieren. Am Gewicht, das der Erstellung einer Disposition, als der zunächst noch formalen Gliederungsleistung des Schülers, zugeschrieben wird, deutet sich das Problem des beschränkten Zugriffes auf das im Aufsatz eigentlich zu Leistende ebenso an, wie im Plädoyer für die Verwendung und Vereinheitlichung von Korrekturzeichen. Es sind Versuche, eine Schülerleistung für formalisierte normierbare Korrektur- und Bewertungszugänge zu öffnen, die in formalen Dimensionen aber gerade nicht aufgehen und bemessen werden soll.

Deshalb zeigen die Philologen auch ein dezidiertes Bemühen, dem Allgemeinbildungsanspruch angemessene Regeln zu finden, wie der deutsche Aufsatz vorzubereiten und zu unterrichten, wie die entstandenen Schülerarbeiten zu beurteilen und die Schülerleistungen einzuschätzen seien. Sie arbeiteten damit an einer Beurteilungs- und Prüfungskultur, zu der eine permissive Grundhaltung sowie die permanente Kritik und Reflexion der eigenen Entscheidungsfindung und des taktvollen Abwägens von Argumenten im Vertrauen darauf gehörten, dass sich Argumentationen im Gebrauch der Sprache bewähren müssten und daher eine Korrektur auf derselben, man kann sagen hermeneutischen Grundlage ebenfalls möglich sei.

„Während der naturwissenschaftliche Unterricht die Natur zum Gegenstand verstandesmäßiger kühler Beobachtung machte, um den Geist in das Verständnis der Gesetzmäßigkeiten und Notwendigkeit einzuführen, und durch diese objektive Behandlung das Gemüt des Schülers von der Natur zu trennen Gefahr läuft, sollte in der deutschen Stunde die innige Verbindung zwischen Gemüt und Natur wieder hergestellt werden. Hier ist die Sonne nicht bloß der Centralkörper, um den sich die Erde dreht, ein ungeheurer Feuerball, der 20 Millionen Meilen von uns absteht, sondern hier ist sie Lebensspenderin, mit der unser Fühlen und Handeln auf das Innigste verbunden ist" (Legerlotz 1899, 25).

Der holistischen Betrachtung der Gegenstände entspricht die ganzheitliche Inanspruchnahme des Schülers und nicht zuletzt soll auch die Beurteilungsweise des Lehrers dem angepasst erfolgen:

„Die meisten Berichte fordern ausdrücklich, daß in der Beurteilung einer Leistung, die an die Kräfte des Schülers so hohe Anforderungen stelle, der Geist der Nachsicht und Milde walten müsse; das Urteil müsse mehr aufklärend und leitend als tadelnd und abweisend sein" (Legerlotz 1900, 138).

Die aus der Diskussion gewonnenen „Leitsätze“ für die Wahl der Themen sowie die Vorbereitung und Beurteilung der deutschen Aufsätze werden der Buchpubli- 
kation von 1900 angehängt. Allein 38 Regeln sind für die Korrektur, Zensur und Rückgabe der Arbeiten aufgestellt:

\section{„Gesichtspunkte für die Beurteilung der Aufsätze“ (Auszug)}

18. Korrektur und Censur müssen sachgemäß und wohlmeinend gehalten sein.

19. Redlichem Fleiß ist stets Anerkennung zu zollen, jedoch nicht auf Kosten der Wahrheit gegenüber der inneren Beschaffenheit der Leistung.

20. Unfleiß, Unredlichkeit, Überhebung und Heuchelei sind streng zu rügen.

21. Persönliche und örtliche Verhältnisse, welche die Leistung eines Schülers erschweren, können auf die Form der Beurteilung mitbestimmend wirken, dürfen aber die Censierung des wirklichen Wertes der Arbeit nicht fälschen.

22. Korrektur und Censur haben nicht nur die Mängel, sondern auch die Vorzüge der Leistung zu berücksichtigen und beide gegeneinander abzuwägen; in zweifelhaften Fällen mag der Geist der Milde den Ausschlag geben.

23. Einige Verstöße gegen die gegenwärtige Rechtschreibung dürfen einem nach Inhalt und Form sonst befriedigenden Aufsatz nicht das Prädikat Ungenügend zuziehen. ${ }^{7}$

24. Tadellose Zeichensetzung verdient Anerkennung, vereinzelte Mängel haben Anspruch auf schonende Beurteilung.

25. Grobe grammatische Fehler fallen schwer in die Wagschale [!] und müssen bei häufiger Wiederkehr Zweifel an der Reife des Schülers erwecken.

26. Unbegründeter Gebrauch von mundartlichen Wörtern und Wendungen von Fremdwörtern und dergl. ist abzulehnen, doch nicht allzu schwer anzurechnen; die Korrektur hat sich vor engherzigen und geschmacklosem Purismus zu hüten.

27. Ästhetische Mängel des Stils wie Plattheit, Schwulst, Weitschweifigkeit u.s.w. sind als solche zu kennzeichnen, doch milde zu beurteilen; Trockenheit und Magerkeit sind nicht zu rügen, Fülle und Schwung dagegen anzuerkennen, doch nicht zu fordern; ein Ansatz zu eigenartigem Stil ist schonend zu behandeln.

28. Logische Mängel des Stils, Unklarheit des Ausdrucks, Verworrenheit in der Entwickelung und Verknüpfung der Gedanken, sind als schwerwiegend anzusehen und können durch bloße grammatische Sprachrichtigkeit und eine gewisse Glätte der Darstellung nicht völlig wettgemacht werden.

29. Von gleichem Gewicht sind logische Mängel in der Stoffgliederung.

30. Hinsichtlich des Inhalts ist eine erschöpfende Behandlung der Gegenstände nicht zu verlangen; der Lehrer hat nur zu fordern, daß die Aufgabe richtig angegriffen und daß die wesentlichen Punkte aufgefunden und sachgemäß bearbeitet seien. Selbständigkeit des Urteils ist anerkennend hervorzuheben.

31. An die Korrektur hat sich ein Gesamturteil anzuschließen, das alle Seiten der Leistung bewertet.

32. Den Schluß hat ein alles zusammenfassendes Prädikat zu bilden; falls Inhalt und Form sich nicht völlig decken, ist das Hauptgewicht auf die reallogische Seite zu legen.

7 Bezugnahme auf die Irritationen im Zuge der Rechtschreibreformen im späten 19. Jahrhundert. 
Auf lange Sicht haben die vorgestellten Formen der Selbstvergewisserung der Praxis der Korrektur und Benotung nicht ausgereicht, um die Kritik am deutschen Aufsatz, dass in ihm etwas abgeprüft werde, was primär in der Schule eben nicht vermittelt wurde und nur denen Vorteile verschaffte, die über den nötigen habituellen Vorsprung ohnehin verfügten, zum Verstummen zu bringen. Es spricht aber für die Qualitäten ihrer Reflexion, dass die Direktoren in einer Zeit, als Bildungsgerechtigkeit und Chancengleichheit, die Selektivität des Schulwesens noch kein vorrangiges Thema professioneller Erörterungen im höheren Schulwesen waren, schon selbst die Grenzen erkannten, an denen die großen Erwartungen an den deutschen Aufsatz als angeblich idealem Prüfmittel bald haltmachen mussten. Einer der Direktoren formulierte es so:

Nächst der Religion ist der deutsche Aufsatz das Gebiet, auf dem das Elternhaus der Arbeit der Schule am meisten hinderlich oder förderlich sein kann. Der Beurteilende wird nicht, wie viele wollen, an den Namen des Verfassers der Arbeit erst dann denken, wenn er das ohne jede Rücksichtnahme gefundene Urteil in sein Notizbuch einträgt; er wird schon vor Beginn der Korrektur der Arbeit sich vergewissern, ob ihr Verfasser aus einer gebildeten Familie stammt, oder ob dessen Vater etwa Briefträger ist und seine Mutter auf dem Markte sitzt und Gemüse verkauft. ${ }^{8}$

Freilich war das 1899 noch erst eine marginale Position. Die umständliche Gegenargumentationen des Hauptreferenten Legerlotz, sein Plädoyer für eine genaue, pädagogisch-wohlwollende Beurteilung jedes Aufsatzes, in der gleichwohl das "thatsächlich Geleistete" maßgeblich für die Bewertung sein sollte (Legerlotz 1899, 93), hinterlässt aus heutiger Perspektive den Eindruck, dass der zitierte Direktor mit seiner Kritik einen wunden Punkt getroffen hatte.

\section{Zur historischen Praxis der Leistungsbewertung im deutschen Abituraufsatz}

Wie nah Historiker*innen der vergangenen Praxis des Bewertens und Prüfens kommen können, ist mit der Frage des Ansatzes zugleich auch eine der Quellenauswahl. ${ }^{9}$ Nur selten sind bisher überlieferte Aufsätze und Aufsatzkorrekturen selbst als Protokolle historischer Prüfungspraxis herangezogen worden (z.B. bei Bölling 2010, 155-180). Zumeist wurde lediglich dem Wandel in den Aufgabenstellungen Beachtung geschenkt. Wo sich Aufsatzkorpora (punktuell, dann aber meist als serielle Quelle) erhalten haben, kann man genauer als an irgendeinem

8 Anonymer Berichterstatter aus Burg bei Magdeburg, zit. nach Legerlotz (1900), 136.

$9 \mathrm{Zu}$ Potentialen und Erträgen praxistheoretischer Forschung in der Erziehungswissenschaft resp. Bildungsgeschichte vgl. Berdelmann u.a. 2019. 
anderen historischen Material erkennen, wie Lehrer/innen tatsächlich die ihnen vorliegenden Aufsätze beurteilten, worauf sie achteten, was sie übersahen. Bis zu einem gewissen Grad ist ersichtlich, nicht nur was, sondern auch wie geprüft wurde, wie Lehrkräfte ihre Schlüsse zogen und das Urteil am Ende der Arbeit tatsächlich zustande kam.

Die für die folgende Betrachtung herangezogenen Abituraufsätze einer Berliner Höheren Schule im Norden (vor) der Stadt entstanden jeweils etwa 40 Jahre vor und 40 Jahre nach den im vorangegangenen Abschnitt wiedergegebenen Diskussionen um die Beurteilung des deutschen Aufsatzes. Hinsichtlich ihrer Materialität lässt sich in den Aufsätzen hochgradige Formstabilität feststellen (vgl. Klinger 2018, v.a. 189-192). Die äußere Erscheinung des deutschen Aufsatzes und die Form, in der er bewertet wurde, blieben über Jahrzehnte beinahe unverändert. Wie bei behördlichen Schreiben nutzt der Schüler nur die rechte Seitenspalte (das Blatt wurde vorher vom Schüler geknickt), er nennt das zu bearbeitende Thema. Lehrer korrigieren mit roter Tinte, machen Randbemerkungen, unterstreichen und korrigieren im Text und geben am Ende eine abschließende Beurteilung, die die Leistung einschätzen und die gefundene Note begründen soll. Das zeigt bereits den hohen Grad und Einfluss eingespielter Formen an, in denen Lehrer/ innen sich bei der Korrektur der Aufsätze bewegten. ${ }^{10}$ Bemerkt wird am Ende immer auch als Vorzensur die Klassenleistung des Schülers. Es stehen in den 1850er-Jahren andere Notenstufen zur Auswahl als dann im 20. Jahrhundert. ${ }^{11}$ 16 Arbeiten zum Thema „In welchem Zusammenhange stehen die Staatsverfassungen der Athener und Spartaner mit der Blüthe und dem Verfall ihrer Macht“ auf 92 teils eng beschriebenen Seiten hatte Lehrer Jung im betrachteten Klassensatz von 1859 zu begutachten. ${ }^{12}$ Am Ende hatte er hier sechsmal auf ,gut', achtmal auf ,befriedigend' und zweimal auf ,nicht befriedigend' befunden. Das theoretisch auch mögliche "vorzüglich“ hat er nicht vergeben. Im Fließtext der Schüleraufsätze markierte er Rechtschreibfehler und nahm Satzumstellungen vor. Er unterstrich dabei einzelne Worte und Passagen (auch doppelt) und nutzte in den Randbemerkungen Symbole wie „?" und „\#“; er schrieb „unklar“, „unüberlegt“, „schlecht ausgedr.[ückt]“, „ohne Sinn“, „zu allgemein“ oder „ungeschickt“,

10 Dass sich ein hohes Maß an Routine bei der Aufsatzbewertung nicht ohne Grund einstellte, veranschaulicht die folgende von Bölling wiedergegebene Selbstauskunft eines Lehrers: „Was [...] wäre eher geeignet, uns den Beruf eines Deutschlehrers [...] verwünschen zu lassen als die Marter, immer wieder und wieder, zehnmal, zwanzigmal, dreißigmal das niederträchtige, beinahe in jedem Satz verfehlte, den hohen Gegenstand kläglich mißhandelnde Zeug lesen und verbessern zu müssen, das der Durchschnittsprimaner über, Wallenstein' oder, Tasso' zu Papier zu bringen imstande ist!“ (Martin Havenstein, Die Dichtung in der Schule, Frankfurt am Main 1925: 19, zit. nach Bölling 2010, 170).

11 Seit 1856 „vorzüglich, gut, befriedigend, nicht befriedigend“, seit 1882 „sehr gut, gut, genügend und nicht genügend" (vgl. Bölling 2010, 39, 97f.)

12 DIPF/BBF/Archiv. GHO 11. 
„Wiederholung“, „störend“, „überflüssig“, „Ausdruck ungeschickt“, „nicht richtig schematisiert", „das hätte bewiesen werden sollen“, „ohne Bedeutung“, „Was heißt das?" „Die ganze Behauptung ist sehr unüberlegt“, „unlogisch“, „sehr ungeschickt", „Die Betrachtung ist sehr schwach“.

Schnell ist zu erkennen: Der Lehrer weist in seinen Randbemerkungen ausschließlich auf Fehler im Aufsatz hin. Es fehlen lobende Bemerkungen irgendwelcher Art oder auch nur Markierungen interessanter Stellen. Nur die oft sehr knappe Abschlussbeurteilung informiert über das positiv Geleistete, wie z.B. in dem gut benoteten Aufsatz des Schülers Wernicke:

„Die Arbeit empfiehlt sich durch klare Auffassung des Themas, durch eingehende Betrachtung der in Frage kommenden Geschichte und durch ihren leichten und fließenden Stil. - Jung. "13

Für eine klare Benennung der Mängel einer Arbeit bestanden gute Gründe, denn Fehleraufzählungen konnten der Lehrkraft etwa als Rechtfertigungsgrundlage gegenüber nachträglich vorgebrachter Kritik dienen. Dennoch wird klar, dass auf die vom Lehrer gewählte Weise die Aufsatzleistung in hohem Maße intuitiv honoriert wurde. Diffusität in der Bewertungspraxis zeigt sich klar auch bei der Behandlung der beiden Aufsätze des Klassensatzes, die am Ende ein „nicht befriedigend" erhielten. Sie wurden zwar zusammen mit den anderen mit Worturteilen versehen, aber erst im Nachhinein benotet, wie die Verwendung kräftigerer roter Tinte, die auch für spätere Einfügungen Verwendung fand, deutlich erkennen lässt (s. Abb. 1). Die Vermutung liegt nahe, dass der Lehrer diese Arbeiten bereits aussortiert hatte, aber erst nach dem Vergleich mit allen anderen Arbeiten die Note einsetzte. Eine der beiden Beurteilungen wurde offensichtlich nach getroffener Entscheidung auch inhaltlich noch so ergänzt, dass die Notengabe stimmiger erschien:

Ursprünglich mit blasser roter Tinte:

„Der Stil entspricht den Anforderungen, der Inhalt zeugt von Unkenntniß der Geschichte und von Unbehülflichkeit im Nachdenken."

Text nach den Ergänzungen (Nachträgliche Einfügungen hier unterstrichen):

„Nicht befriedigend. Der Stil entspricht den Anforderungen nur unvollkommen, der Inhalt zeugt von Unkenntniß der antiken Geschichte und von Unbehülflichkeit im Nachdenken in einem solchen Grade, daß die Arbeit nicht für befriedigend gelten kann. ${ }^{.14}$

13 DIPF/BBF/Archiv: GHO 11, Bl. 4.

14 Aufsatz des Schülers Laue. In: DIPF/BBF/Archiv. GHO 11, Bl. 11. 


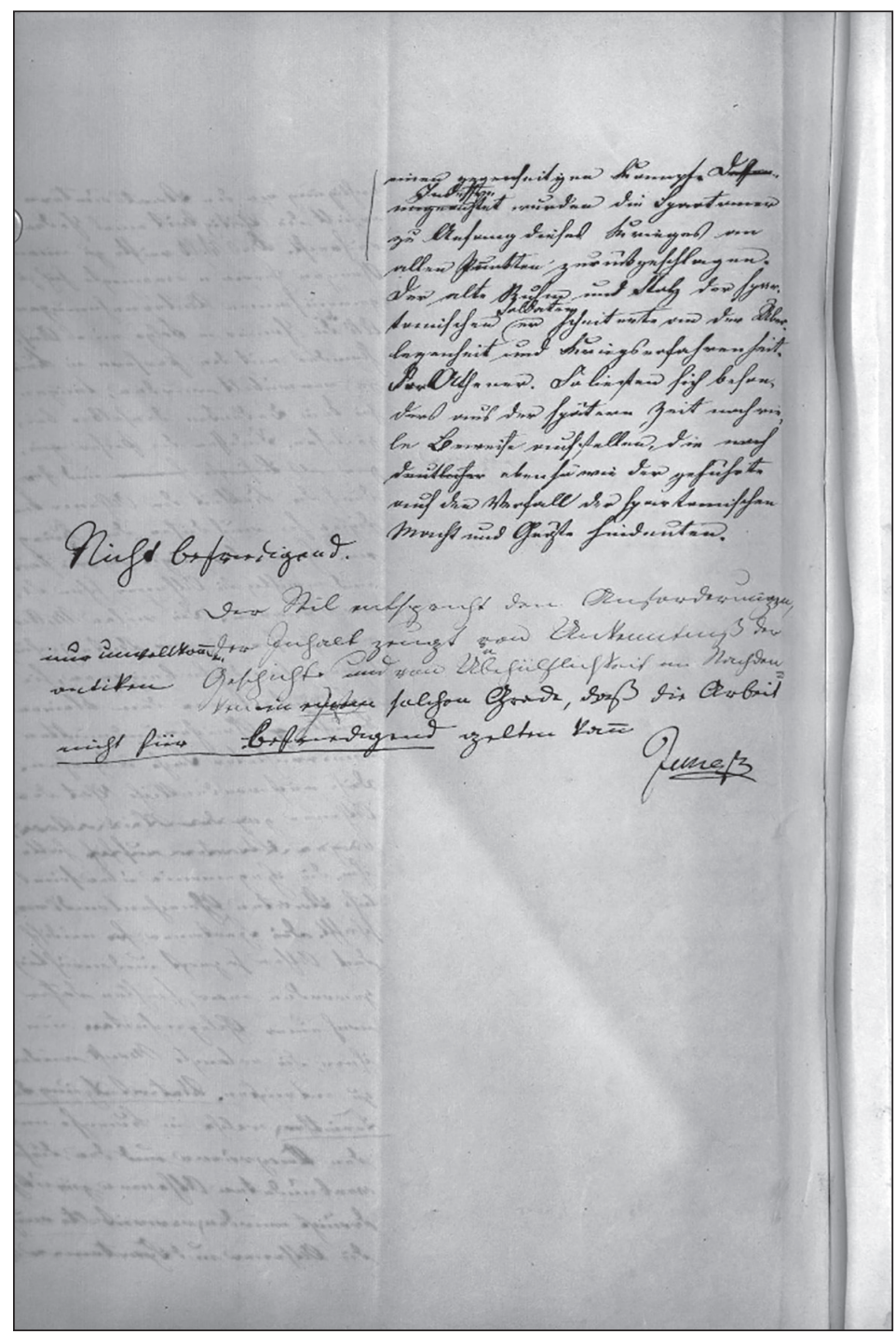

Abb. 1: Beurteilung eines Schüleraufsatzes mit nachträglichen Änderungen und Benotung (1859).

Die Formulierung, der sich der Lehrer bei der Beurteilung bediente, war an die individuelle Qualität der Schülerarbeit auf eine Weise gebunden, die dehnbar und durch Ergänzungen akzentuierbar war. Die Note hätte auch anders ausfallen können. Nicht die Einzelleistung, sondern erst der Vergleich mit anderen Arbeiten brachte die Entscheidung. 


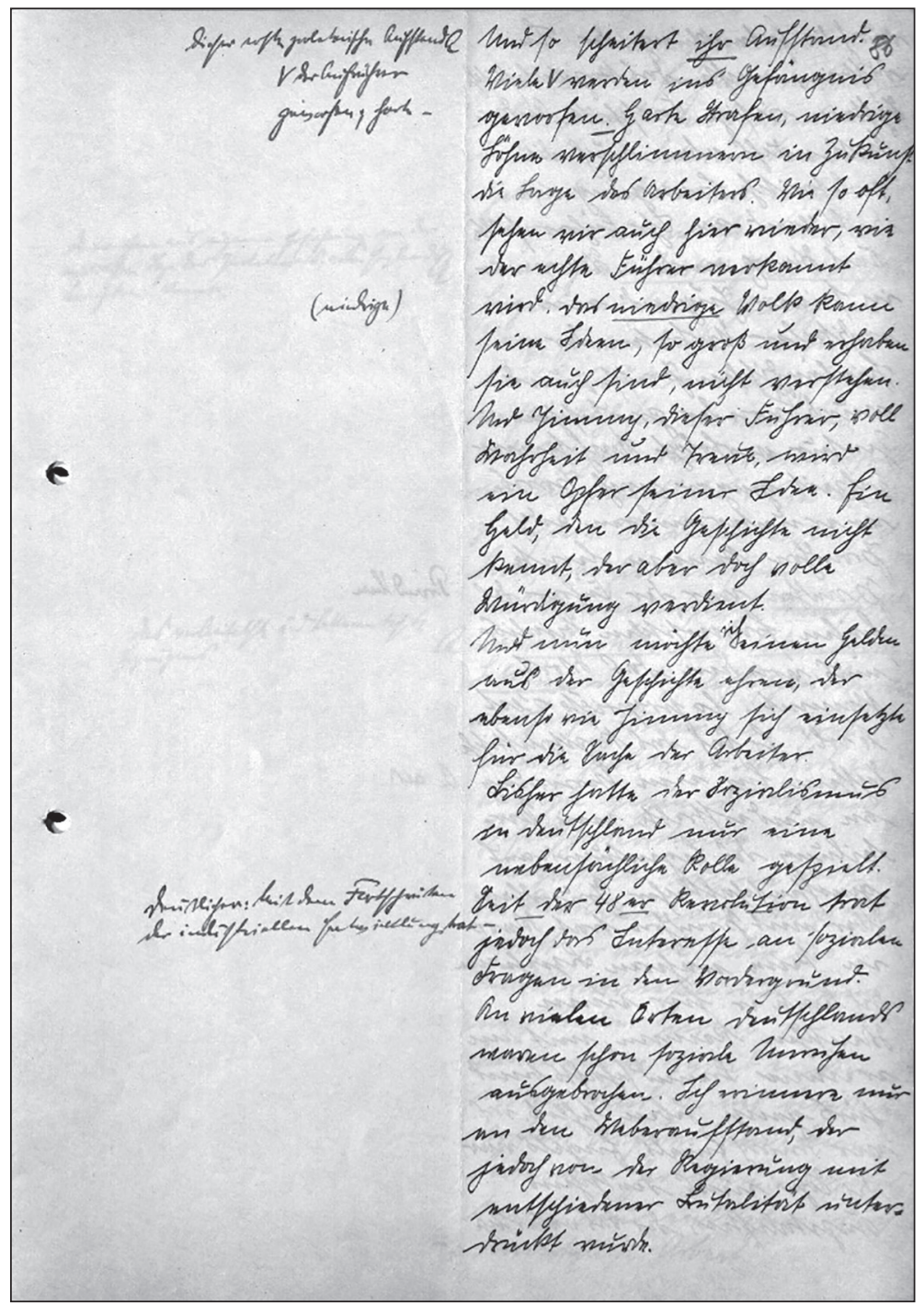

Abb. 2: Randbemerkungen an einem Ostern 1932 mit "gut" beurteilten Aufsatz. ${ }^{15}$

Im Verlauf der Jahrzehnte, so lässt sich allgemein feststellen, werden die Korrekturen und Begründungen in Randbemerkungen sowie die Beurteilungen am Ende der Abituraufsätze ausführlicher. Das kann als Reaktion auf die der Prüfungsform 
eigenen Indifferenzen interpretiert werden und zugleich als Indiz für eine gesteigerte Begründungsverpflichtung, da die Abiturnoten und damit auch die Aufsatznote nun tatsächlich und nicht mehr nur symbolisch selektionsrelevant und damit karriereentscheidend sein konnten. Den Arbeiten selbst ist der gesteigerte Aufwand anzusehen, den die Korrektur von Rechtschreibfehlern und die Umstellung von Formulierungen in den Randbemerkungen gekostet haben. Zwar hat sich in späteren Stichproben der Trend nicht verloren, im Einzelnen nur Fehler, nicht aber die gezeigten Leistungen zu markieren. Auffällig ist jedoch, dass nun in den Gedankengang der Schülerinnen und Schüler selbst nicht mehr so unbefangen eingegriffen wurde wie 80 Jahre zuvor. Offenbar hatte eine Auffassung von Individualität des Schülers und Authentizität seines Ausdrucks die Praxis erreicht, zu der es gehörte, dass man der Schülerargumentation mit größerem Respekt begegnete, sie nicht mehr wie einen Rechtschreibfehler korrigierte, sondern zunächst gelten ließ. Stattdessen werden Befragungen und Einschätzungen gängig.

Korrekturen und Randbemerkungen (Unterstreichungen) an einem Ostern 1932 mit "gut" beurteilten Aufsatz (Ausschnitte) ${ }^{16}$

\begin{tabular}{|c|c|c|}
\hline Schüler schreibt & $\begin{array}{l}\text { Lehrer korrigiert im } \\
\text { Fließtext }\end{array}$ & Lehrer bemerkt am Rand \\
\hline $\begin{array}{l}\text { Und so scheitert ihr } \\
\text { Aufstand. Viele werden ins } \\
\text { Gefängnis geworfen. }\end{array}$ & $\begin{array}{l}\text { Und so scheitert ihr } \\
\text { Aufstand. Viele werden ins } \\
\text { Gefängnis geworfen. Harte } \\
\text { Strafen }\end{array}$ & $\begin{array}{l}\text { R Und so scheitert dieser } \\
\text { erste proletarische Auf- } \\
\text { stand. Viele der Anführer } \\
\text { werden ins Gefängnis } \\
\text { geworfen; harte - }\end{array}$ \\
\hline Das niedrige Volk & & (niedrige) \\
\hline $\begin{array}{l}\text { Seit der 48er Revolution } \\
\text { trat jedoch das Interesse } \\
\text { an sozialen Fragen in den } \\
\text { Vordergrund. }\end{array}$ & $\begin{array}{l}\text { Seit der 48er Revolution } \\
\text { trat jedoch das Interesse } \\
\text { an sozialen Fragen in den } \\
\text { Vordergrund. }\end{array}$ & $\begin{array}{l}\text { Deutlicher: mit dem Fort- } \\
\text { schreiten der industriellen } \\
\text { Entwicklung trat - }\end{array}$ \\
\hline $\begin{array}{l}\text { Er sah das kapitalistische } \\
\text { System für eine Verirrung } \\
\text { an }\end{array}$ & $\begin{array}{l}\text { Er sah das kapitalistische } \\
\text { System als eine Verirrung an }\end{array}$ & \\
\hline $\begin{array}{l}\text { da er [Engels] ihm [Marx] } \\
\text { die Wirklichkeit berichtete }\end{array}$ & $\begin{array}{l}\text { da er ihm aus eigener Er- } \\
\text { fahrung von der wirklichen } \\
\text { Lage des Proletariats, vor- } \\
\text { neml. Englands berichten } \\
\text { konnte. }\end{array}$ & \\
\hline Anfangs glaubte Marx & Anfangs dachte Marx & \\
\hline
\end{tabular}

16 DIPF/BBF/Archiv: GHO 134, Bl. 84-89. 
Überdies fällt bei Aufsätzen aus der späten Weimarer Republik ins Auge, dass in den Gesamturteilen viel stärker als zuvor Schülerinnen und Schüler in ihrer Persönlichkeit Beachtung finden. ${ }^{17}$ Der Lehrer nimmt in den Beurteilungen etwa Einschätzungen der Selbstständigkeit des Denkens eines Schülers vor, auch bezieht er sich auf ihm bekannte Eigenarten des Schülers und auf dessen Art, sich zum Aufsatzgegenstand ins Verhältnis zu setzen. So wird einem mit gut bewertetem Schüler etwa eine "gradlinige Gewaltsamkeit des Denkens" ${ }^{\text {"18 }}$ attestiert und eine „Fähigkeit, Gedanken auf das wesentliche zu reduzieren“ (ebd.). Demgegenüber mangelt es nach Einschätzung des Lehrers einem anderen an der Fähigkeit, „über relativ einfache Fragen nachzudenken“ ${ }^{19}$ Auch eine im Aufsatz gezeigte „Begeisterung" des Schülers für sein Thema wird im Sample von 1930 mehrfach gewürdigt und entschied in diesem Fall ausdrücklich über die Notenenverbesserung.

\section{Fazit}

Mit dem Bedeutungsgewinn schulischer Prüfungen für den Lebenslauf einzelner Schüler*innen wurden in den Regularien, die den Universitätszugang und damit die Elitereproduktion im 19. Jahrhundert zunehmend steuerten, immer genauer die Art und Weise der Durchführung der Abiturprüfung festgelegt. Der deutsche Aufsatz war von diesem Prozess nicht ausgenommen. Einerseits erhöhte sich sein Gewicht durch die offensichtlichen Anschlussmöglichkeiten an das Allgemeinbildungskonzept des Nationalstaates, andererseits zeigt sich an ihm, dass das holistische Beurteilungssystem gerade zur Forderung nach spezifischen, individuell zurechenbaren und überindividuell vergleichbaren Leistungsbewertungen in Opposition stand. Die Beurteilung des deutschen Abituraufsatzes erfolgte allen Bemühungen um gerechte Beurteilungskriterien zum Trotz in recht offenen Kommentierungsund Bewertungspraktiken durch die Lehrer. Der deutsche Aufsatz war zudem wenig auf Fachlichkeit bezogen, allenfalls im Sinne einer nationalen Literaturgeschichte kam diese ansatzweise zur Geltung. Es hatten sich dagegen Beobachtungsfelder für Texte und Personen herausgebildet und Konventionen, wie kommentiert und beurteilt werden sollte. In welchem Verhältnis zueinander sie aber in der Endbenotung standen, blieb der Abwägung durch den Lehrer überlassen.

Hier setzte schließlich die Kritik an der Subjektivität der Benotung des Aufsatzes und seiner problematischen Sonderstellung im schulischen Prüfungswesen an, die seit den 1960er-Jahren, verstärkt dann in den Strukturreformen des Bildungswe-

17 Die in dieser Passage referierten Quellen und Aussagen beruhen auf Recherchen von Kathrin Berdelmann, der ich für die Erlaubnis zur Wiedergabe herzlich danke.

18 DIPF/BBF/Archiv: GHO 86, Bl 6.

19 DIPF/BBF/Archiv: GHO 86, Bl. 16. 
sens, grundlegende Veränderungen nach sich zog. Gerade weil der deutsche Aufsatz als Ausdruck der Bildung einer Person, als gänzlich individueller Ausdruck der Persönlichkeit galt, fiel er schließlich den Bemühungen um „Objektivierung“ und Standardisierung der Schülerbeurteilung zum Opfer. Dass damit auch eine Kompetenz der taktvoll-angemessenen Bewertung an Bedeutung einbüßte, steht auf einem anderen Blatt.

\section{Quellen und Literaturverzeichnis}

\section{Quellen}

Bibliothek für Bildungsgeschichtliche Forschung des DIPF | Leibniz-Institut für Bildungsforschung und Bildungsinformation

DIPF/BBF/Archiv: GHO 11: Reifeprüfungsarbeiten Deutsch, Ostern 1859

DIPF/BBF/Archiv: GHO 86: Reifeprüfungen Michaelis 1931.

DIPF/BBF/Archiv: GHO 134: Reifeprüfungen Ostern 1932.

\section{Gedruckte Quellen}

Apelt, O. (1907): Der deutsche Aufsatz in der Prima des Gymnasiums. Ein historisch-kritischer Versuch. Leipzig und Berlin: B. G. Teubner.

Cirkularerlaß, betreffend Ordnung der Entlassungsprüfungen an den höheren Schulen vom 27.05.1882 (Circularerlass 1882). In: Centralblatt für die gesammte Unterrichts-Verwaltung in Preußen. Herausgegeben in dem Ministerium der geistlichen, Unterrichts- und Medizinal-Angelegenheiten. Jahrgang 1892, 365-415.

Hense; Meyer (1882): Über den deutschen Unterricht an höheren Lehranstalten mit eingehnder Berücksichtigung der Lehrpläne vom 31. März 1882. In: Verhandlungen der Direktoren-Versammlungen in den Provinzen des Königreichs Preußen seit dem Jahre 1879. 33. Band: Zweiundzwanzigste Direktoren-Versammung in der Provinz Westfalen 1879. Berlin: Weidmannsche Buchhandlung. 46-141.

Michael, B.; Schepp, H.-H. (Hrsg.)(1973): Politik und Schule von der Französischen Revolution bis zur Gegenwart. Eine Quellensammlung zum Verhältnis von Gesellschaft, Schule und Staat im 19. und 20. Jahrhundert. Band 1, Frankfurt am Main: Athenäum Fischer Taschenbuch Verlag, 414-419.

Ihm, G. (1889): Korrektur und Rückgabe der deutschen Aufsätze. In: Gymnasium. Zeitschrift für Lehrer an Gymnasien und verwandten Unterrichtsanstalten - 7. Jahrgang, Heft 7, 73-78.

Legerlotz, G. (1899): Nach welchen Gesichtspunkten ist der deutsche Aufsatz in den oberen Klassen zu wählen, vorzubereiten und zu beurteilen?. In: Verhandlungen der Direktoren-Versammlungen in den Provinzen des Königreichs Preußen seit dem Jahre 1879. 56. Band: Achte DirektorenVersammlung in der Provinz Sachsen, 1-109.

Legerlotz, G. (1900): Der deutsche Aufsatz auf der Oberstufe der höheren Lehranstalten. Berlin: Weidmannsche Buchhandlung.

Neigebaur, J. D. F. (1835): Die preussischen Gymnasien und höheren Bürgerschulen. Eine Zusammenstellung der Verordnungen, welche den höheren Unterricht in diesen Anstalten umfassen. Berlin, Posen, Bromberg: Mittler.

Ordnung der Reife- und Abschlußprüfungen 1892. In: Centralblatt für die gesammte UnterrichtsVerwaltung in Preußen. Herausgegeben in dem Ministerium der geistlichen, Unterrichts- und Medizinal-Angelegenheiten. Jahrgang 1892, 280-351.

Schultze, F. (1831): Die Abiturienten-Prüfung, vornehmlich im Preußischen Staate. A. UrkundenSammlung. Liegnitz und Halle: Eduard Anton. 
Schröer \& Dolega (1891): Der deutsche Unterrich in der Sekunda und Prima. In: Verhandlungen der Direktoren-Versammlungen in den Provinzen des Königreichs Preußen seit dem Jahre 1879. 36. Band: Neunte Direktoren-Versammung in der Provinz Posen 1891. Berlin: Weidmannsche Buchhandlung, 1-74.

\section{Literatur}

Berdelmann, K.; Fritzsche, B.; Rabenstein, K.; Scholz, J. (Hrsg.) (2019): Transformationen von Schule, Unterricht und Profession. Erträge praxistheoretischer Forschung. Wiesbaden: Springer VS (Sabine Reh zum 60. Geburtstag).

Berdelmann, K.; Reh, S.; Scholz, J. (2018): Wettbewerb und Ehrtrieb. Die Entstehung des LeistungsDispositivs im Schulwesen um 1800. In: S. Reh, \& N. Ricken (Hrsg.): Leistung als Paradigma. Zur Entstehung und Transformation eines pädagogischen Konzeptes, Wiesbaden: Springer-VS, 137-163.

Bölling, R. (2010): Kleine Geschichte des Abiturs. Paderborn, München, Wien, Zürich: Ferdinand Schöning.

Jacobi, J. (2007): Lektüre schützt vor Neuentdeckung: Zur Funktion der historischen Bildungsforschung für die Erziehungswissenschaft. In: M. Brumlik \& H. Merkens (Hrsg.): bildung - macht - gesellschaft. Beiträge zum 20. Kongress der Deutschen Gesellschaft für Erziehungswissenschaft, Opladen und Farmington Hills: Verlag Barbara Budrich, 43-58.

Jeismann, K.-E. (2. Aufl. 1996): Das preußische Gymnasium in Staat und Gesellschaft. Band 2: Höhere Bildung zwischen Reform und Reaktion 1717-1859. Stuttgart: Klett-Cotta.

Jeismann, K.-E. (1999): Zur Professionalisierung der Gymnasiallehrer im 19. Jahrhundert. In: H. J. Apel, K.-P. Horn, P. Lundgreen \& U. Sandfuchs (Hrsg.): Professionalisierung pädagogischer Berufe im historischen Prozeß, Bad Heilbrunn: Verlag Julius Klinkhardt, 59-79.

Kämper-van den Boogaart, M. (2012): Der deutsche Aufsatz und das Abitur. Was man vielleicht aus der Geschichte lernen könnte. In: H. Feilke, J. Köster \& M. Steinmetz (Hrsg.): Textkompetenzen in der Sekundarstufe II. Stuttgart: Fillibach bei Klett, 41-62.

Klinger, K. (2018): Das Abitur - Eine Akte. Zu einer Historischen Praxeologie des Abiturs. In: Sektion Historische Bildungsforschung der Deutschen Gesellschaft für Erziehungswissenschaft (Hrsg.): Jahrbuch für Historische Bildungsforschung 2018. Band 23. Schwerpunkt: Scheinbarer Stillstand - Pädagogische Diskurse und Entwicklungen in den Achtzigerjahren, Bad Heilbrunn: Verlag Julius Klinkhardt, 172-204.

Kluchert, G. (2014): Die Gymnasiallehrer - Kontinuität und Wandel in beruflichem Selbstverständnis und Handeln. In: Ritzi, Christian; Tosch, Frank (Hrsg.): Gymnasium im strukturellen Wandel. Befunde und Perspektiven von den preußischen Reformen bis zur Reform der gymnasialen Oberstufe, Bad Heilbrunn: Verlag Julius Klinkhardt, 35-63.

Müller, D. K. (1977): Sozialstruktur und Schulsystem. Aspekte zur Theorie und Praxis der Schulorganisation im 19. Jahrhundert. Göttingen und Zürich: Vandenhoeck \& Rupprecht (Inaugiuraldissertation zur Erlangung des Doktorgrades der Philosophischen Fakultät der Johann Wolfgang GoetheUniversität zu Frankfurt am Main).

Reh, S.; Kämper-van den Boogaart, M. \& Scholz, J. (2017): Eine lange Geschichte: Der deutsche Abituraufsatz als „Gesammtbildung des Examinanden“. Prüfungspraxis und Lehrerkommentare von Abituraufsätzen in den 1950er Jahren. In: Zeitschrift für Pädagogik 3, 280-298.

Scholz, J. \& Reh, S. (2016): Auseinandersetzungen um die Organisation von Schulklassen. Verschiedenheit der Individuen, Leistungsprinzip und die moderne Schule um 1800. In: C. Groppe, G. Kluchert $\&$ E. Matthes (Hrsg.): Bildung und Differenz. Historische Analysen zu einem aktuellen Problem, Wiesbaden: Springer-VS, 93-113.

Schubring, G. (1991): Die Entstehung des Mathematiklehrerberufs im 19. Jahrhundert. Studien und Materialien zum Prozeß der Professionalisierung in Preußen (1810-1870). Weinheim: Deutscher Studien Verlag. 